\title{
STAR FORMATION HISTORY OF A YOUNG SUPER-STAR CLUSTER IN NGC 4038/39: DIRECT DETECTION OF LOW-MASS PRE-MAIN SEQUENCE STARS
}

\author{
Julia Greissl ${ }^{1}$, Michael R. Meyer ${ }^{1,2}$, Micol H. Christopher ${ }^{3}$, AND Nick Z. Scoville ${ }^{3}$ \\ ${ }^{1}$ Steward Observatory, University of Arizona, Tucson, AZ 85721, USA; jgreiss1@ as.arizona.edu \\ ${ }^{2}$ Institute for Astronomy, ETH Zürich, Wolfgang-Pauli-Str. 27, 8093, Zürich, Switzerland \\ ${ }^{3}$ California Institute of Technology, Pasadena, CA 91125, USA \\ Received 2009 February 25; accepted 2010 January 11; published 2010 February 2
}

\begin{abstract}
We present an analysis of the near-infrared spectrum of a young massive star cluster in the overlap region of the interacting galaxies NGC 4038/39 using population synthesis models. Our goal is to model the cluster population as well as provide rough constraints on its initial mass function (IMF). The cluster shows signs of youth, such as thermal radio emission and strong hydrogen emission lines in the near-infrared. Late-type absorption lines are also present which are indicative of late-type stars in the cluster. The strength and ratio of these absorption lines cannot be reproduced through either late-type pre-main sequence (PMS) stars or red supergiants alone. Thus, we interpret the spectrum as a superposition of two star clusters of different ages, which is feasible since the $1^{\prime \prime}$ spectrum encompasses a physical region of $\approx 90 \mathrm{pc}$ and radii of super-star clusters (SSCs) are generally measured to be a few parsecs. One cluster is young ( $\leqslant 3 \mathrm{Myr})$ and is responsible for part of the late-type absorption features, which are due to PMS stars in the cluster, and the hydrogen emission lines. The second cluster is older (6 Myr-18 Myr) and is needed to reproduce the overall depth of the late-type absorption features in the spectrum. Both are required to accurately reproduce the near-infrared spectrum of the object. Thus, we have directly detected PMS objects in an unresolved SSC for the first time using a combination of population synthesis models and PMS tracks. This analysis serves as a testbed of our technique to constrain the low-mass IMF in young SSCs as well as an exploration of the star formation history of young UC H II regions.
\end{abstract}

Key words: galaxies: individual (NGC 4038, NGC 4039) - galaxies: interactions - stars: luminosity function, mass function - stars: pre-main sequence

\section{INTRODUCTION}

The advent of the Hubble Space Telescope (HST) has made it possible to study in detail nearby starburst galaxies with star formation rates orders of magnitude higher than the Milky Way (e.g., M82, Henize 2-10, and NGC 5253) and many are now known to host massive $\left(10^{5}-10^{6} M_{\odot}\right)$ young super-star clusters (SSCs). These clusters are thought to mirror a mode of star formation that was ubiquitous in the early universe (Leitherer 2001) as evidenced by the fact that star formation rates of galaxies at high redshift are very similar to those in local starbursts (Steidel et al. 1996).

SSCs represent an extreme form of star formation that cannot be studied in the Milky Way. The closest analog is R136 in the LMC with a mass of $10^{4.5} M_{\odot}$ (Massey \& Hunter 1998) though the Galaxy also hosts some clusters with masses greater than $10^{4} M_{\odot}$ (e.g., Westerlund 1 and NGC 3603). Massive young clusters have been of particular interest in the search for initial mass function (IMF) variations. Since the local stellar IMF (within $1 \mathrm{kpc}$ ) appears to be universal (Meyer et al. 2000), we are forced to expand the search for IMF variations to more extreme regions of star formation. SSC often form in the intense radiation environment of nuclear starbursts and interacting galaxies. Thus, they represent ideal objects to search for IMF variations with initial conditions, such as metallicity and formation environment. Many young massive clusters with ages $\leqslant 40 \mathrm{Myr}$ have varying mass-to-light ratios (e.g., NGC 1705-1, Smith \& Gallagher 2001; NGC 1569-B, Anders et al. 2004), which have often been interpreted as changes in the mass function. The mass-to-light ratios are determined through dynamical mass estimates however, which assume that clusters are in virial equilibrium. Goodwin \& Bastian (2006) have shown that this assumption can be faulty in young clusters due to the effects of gas expulsion. The mass-to-light ratio of M82-F however, a massive cluster in the prototypical starburst M82 with an age of 40-60 Myr, remains inconsistent with a Kroupa (2001) IMF (McCrady et al. 2005). Mass-to-light ratios are at best an indirect way to determine the IMF of a young cluster because the whole stellar mass range is represented by one mass bin. A more direct method of detecting stellar populations in unresolved SSC is needed to provide confirmation of the shape of their IMF.

Star clusters are dominated dynamically by their low-mass content. Yet, most of the light in young clusters is seen through their massive stars. To understand whether a young SSC will remain bound or disperse, it is important to get a direct census of low-mass stars. In addition, differences in the IMF of SSCs are most easily detected in low-mass stars, since the characteristic mass in both a Kroupa (2001) and Chabrier (2003) IMF is around $0.5 M_{\odot}$. Even small variations in the IMF of a young SSC may make the difference between a bound and an unbound cluster. The difference between a Salpeter (1955) and a Chabrier (2003) IMF accounts for a factor of 2 in the mass of low-mass stars, for example.

The Antennae (NGC 4038/9) are the nearest (distance = $19.2 \mathrm{Mpc}, 1^{\prime \prime} \approx 93 \mathrm{pc}$; Whitmore et al. 1999) pair of merging spiral galaxies. They contain up to a thousand young star clusters. These clusters have been the target of a multitude of studies at different wavebands (e.g., Whitmore et al. 1999; Neff \& Ulvestad 2000; Brandl et al. 2005). Whitmore \& Schweizer (1995) identified a large population of SSC in the optical using HST WFPC2 images. The faintest and reddest of these sources are revealed to be bright near-infrared and mid-infrared emitters and contain the youngest clusters (Snijders et al. 2006; Wang et al. 2004; Brandl et al. 2005). Radio images by Neff 
Table 1

Archival Data of Cluster 89/90

\begin{tabular}{lcccccccc}
\hline \hline R.A. (J2000.0) & Decl. (J2000.0) & $F_{4 \mathrm{~cm}}(\mu \mathrm{mJy})^{\mathrm{a}}$ & $F_{6 \mathrm{~cm}}(\mu \mathrm{mJy})$ & $m_{V}{ }^{\mathrm{b}}$ & $m_{I}$ & $m_{J}{ }^{\mathrm{c}}$ & $m_{H}{ }^{\mathrm{d}}$ & $m_{K}$ \\
\hline $12: 01: 54.58$ & $-18: 53: 03.42$ & 1957 & 2316 & 19.07 & 18.40 & 15.05 & 14.71 & 14.27 \\
\hline
\end{tabular}

Notes.

${ }^{\text {a }}$ From Neff \& Ulvestad 2000

${ }^{\mathrm{b}}$ From Whitmore \& Zhang 2002.

${ }^{\text {c }}$ From Brandl et al. 2005 in the Two Micron All Sky Survey (2MASS) system.

${ }^{\mathrm{d}}$ From 2MASS.

\& Ulvestad (2000), which detect sources with purely thermal nebular emission (i.e., $\alpha_{4 \mathrm{~cm}-6 \mathrm{~cm}} \geqslant-0.4$ ) due to massive stars in the clusters, provide additional evidence for the youth of these objects. Sources with non-thermal radio emission likely contain supernova remnants and are thus older.

This paper expands on a method introduced in Meyer \& Greissl (2005) to constrain the low-mass stellar content in young SSC using late-type absorption features in the near-infrared. For young clusters, most low-mass objects are still on the pre-main sequence (PMS) and are thus orders of magnitude brighter than their main sequence counterparts. Thus, they can be detected in high signal-to-noise ratio $(\mathrm{S} / \mathrm{N})$ spectra of young SSC. We focus our analysis on one massive young cluster in the Antennae.

Cluster 89/90 (designation of Whitmore \& Schweizer 1995), the cluster targeted in this study, was first surveyed by Whitmore \& Schweizer (1995) using WFPC2 on the HST. It is the brightest near-infrared cluster in NGC 4038/9 as well as the second brightest thermal radio source at 4 and $6 \mathrm{~cm}$. In addition, Snijders et al. (2006) observed the cluster in the mid-infrared and found strong Ne II 12.8 and Ne III $15.5 \mu \mathrm{m}$ emission as well as polycyclic aromatic hydrocarbon emission at $11.25 \mu \mathrm{m}$. These features are an indication of the presence of hot stars (Mirabel et al. 1998). See Table 1 for a list of broadband magnitudes for cluster 89/90 obtained from archival data.

In the following sections, we present a $K$ - and $H$-band spectrum of cluster $89 / 90$ and model its underlying population. Section 2 details the observations and data reduction. Section 3 gives an overview of the method used to model the spectrum of the cluster. The analysis of the spectrum, which constrains the population of the cluster, is presented in Section 4. Section 5 contains the results of the analysis, as well as its limitations. Section 6 places our analysis in the context of previous work. Section 7 details our conclusions.

\section{OBSERVATIONS AND DATA REDUCTION}

$H$ - and $K$-band spectra of cluster $89 / 90$ were obtained with NIRSPEC (McLean et al. 1998) on Keck in 2003 February using the $42^{\prime \prime}$ slit with a scale of $0^{\prime \prime} 144$ pixel $^{-1}$. The spectrum was observed as part of a larger data set of Antennae clusters which are described in detail in Christopher (2008). For the $H$-band spectra, we used the N5 filter, covering 1.54-1.83 $\mu \mathrm{m}$. The $K$-band spectrum was obtained with the N7 filter covering 2.05-2.47 $\mu \mathrm{m}$. The $K$ band seeing during the observations was $\approx 0$.5 and stable throughout the night; accordingly, the 0 '.57 wide slit was used in both bands. The spectra were obtained with a total integration time of $900 \mathrm{~s}(3 \times 300 \mathrm{~s})$ in both the $H$ - and the $K$-band at an air mass of $\approx 1.3$. The object was offset along the slit in successive frames to allow for sky subtraction. Since SSC can often have a wealth of small-scale structure, we were careful to make sure the sky-subtracted images contained no significant galactic background contamination. After sky subtraction, the background had no identifiable shape and the noise in the background was dominated by instrumental noise. We did not see detectable $\mathrm{CO}$ absorption outside of our cluster in the spectrum which would have affected our analysis. Calibration data were obtained including a flat field and appropriate dark frame as well as neon and argon arc lamp spectra to use for wavelength calibration. To correct for telluric absorption, we used a G2V star in the $H$ band and an A0V star in the $K$ band. To ensure the best telluric correction, we observed the standard stars within 0.05 air mass of the target. Flat fielding and cosmic ray removal were performed on each spectrum using standard IDL procedures.

NIRSPEC spectra have spatial and spectral distortions that must be removed during the reduction process. The spatial distortion corrections were calculated by measuring the position of the brightest calibrator spectra at multiple positions in the slit. The traces of these sources were fit with polynomials to determine the spatial distortion correction. This routine was modified from the REDSPEC ${ }^{4}$ package written by Lisa Prato. For wavelength calibration, we used an argon arc lamp in the $H$ band and a neon arc lamp in the $K$ band. The reduction of the calibrator stars was carried out in the same manner. To determine the atmospheric calibration, we applied template spectra using Meyer et al. (1998) in the $H$ band and Wallace \& Hinkle (1997) in the $K$ band smoothed to the resolution of our spectra $(R \approx 1100-1500)$. The calibrator stars were largely featureless, except for $\mathrm{Br} \gamma$ absorption in the $K$ band, which was not well matched by the template and removed independently. Stellar absorption centers were measured in both the template and the calibrator spectrum to remove any spectral offset. The $H$-band template spectra covered the entire wavelength range of the $H$-band atmospheric calibrator observations; for the $K$ band, the template spectra ended at $2.4 \mu \mathrm{m}$, while our observations extended redward to $2.47 \mu \mathrm{m}$. We assumed a featureless blackbody for the template spectra from 2.4 to $2.47 \mu \mathrm{m}$. We examined the final telluric spectrum to ensure that there were no mismatches between the G2V standard and the solar spectrum particularly around the $\mathrm{Mg}$ absorption line at 1.71 $\mu \mathrm{m}$ which could have affected our analysis. The atmospheric calibration was calculated by dividing the calibrator spectrum by the shifted template spectrum and normalizing the result at the center of the band. Any spectral offset between the atmospheric correction and the source spectra were measured by comparing the location of atmospheric absorption features and applying an offset to the atmospheric correction if necessary. Typical offsets were less than 0.1 pixels. The atmospheric correction removes both the effects of the atmosphere and variations in NIRSPEC system throughput as a function of wavelength. The final spectrum was extracted from the atmospherically calibrated composite exposure with an aperture size of $5 \times$ the seeing.

\footnotetext{
4 See http://www2.keck.hawaii.edu/inst/nirspec/redspec/index.html
} 

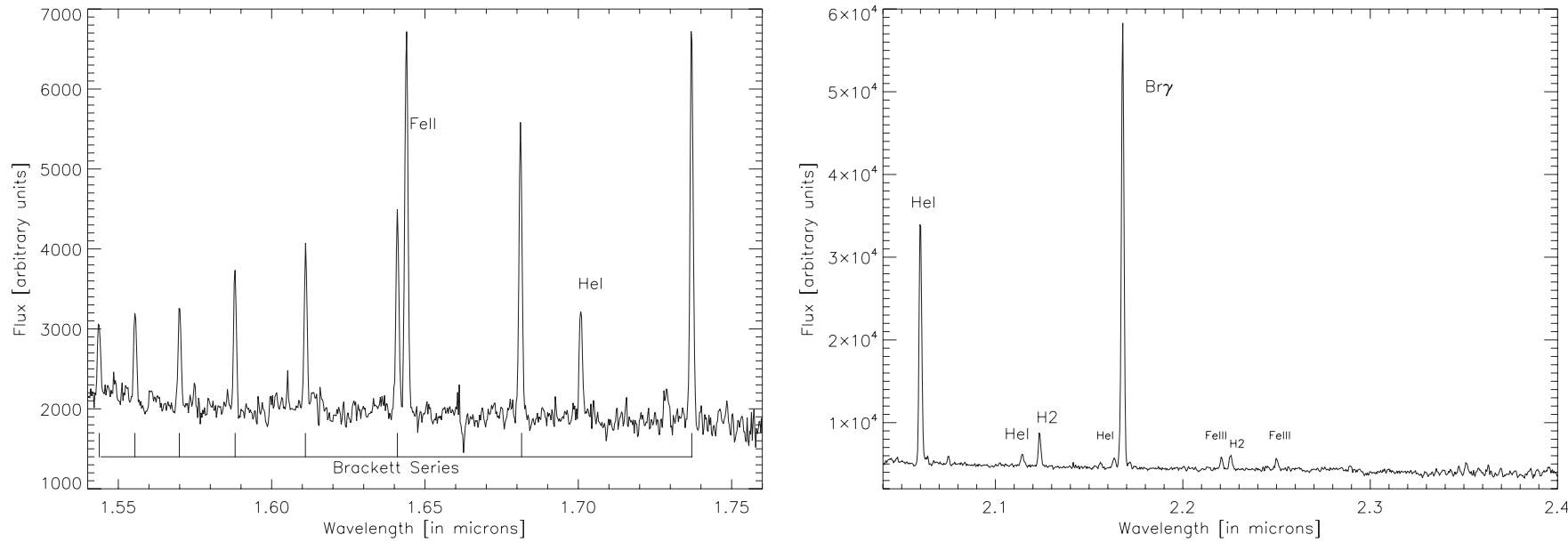

Figure 1. NIRSPEC $H$ - and $K$-band spectrum of cluster $89 / 90$. Emission lines which are indicative of the youth of the cluster are marked. The data have been shifted to rest-frame wavelengths.

Figure 1 shows the final extracted $H$ - and $K$-band spectrum of cluster $89 / 90$.

\section{MODELING THE NEAR-INFRARED SPECTRUM OF UNRESOLVED STAR CLUSTERS}

We model the spectra of young SSCs in the near-infrared incorporating the fact that at young ages low-mass stars are still on the PMS. For objects on the PMS, we use our own synthesis code combined with a set of PMS tracks (Siess et al. 2000). For any objects not on the PMS, we use the STARBURST99 (S99) population synthesis models (Leitherer et al. 1999) which are designed to accurately reproduce spectrophotometric properties of starbursts and SSC but do not incorporate any PMS tracks. For our S99 models, we used the included Padova tracks with AGB stars and the atmospheres used were Pauldrach/ Hillier (Vazquez \& Leitherer 2005). The final model has two separate components, one stellar component as well as a nebular component due to thermal free-free and free-bound emission from hot stars in the cluster. In Section 3.1, we describe the inputs and parameters of the stellar model, while Section 3.2 details the constraints we can place on the nebular emission in clusters empirically.

\subsection{Stellar Component}

The stellar spectrum of our clusters is modeled in the following way (see also Meyer \& Greissl 2005). To determine the most massive star still on the PMS at a given age, we use the tracks of Siess et al. (2000). At ages of 1 and $3 \mathrm{Myr}$, this corresponds to $7 M_{\odot}$ and $5 M_{\odot}$, respectively. An appropriate PMS mass-luminosity relationship for this age is then assumed (Siess et al. 2000). Stars above this limiting mass are modeled through S99. This includes the main sequence as well as the postmain sequence. We use instantaneous burst models assuming a Salpeter (1955) IMF above the PMS cutoff combined with the evolutionary models of the Padova group. The mass of the PMS and $\mathrm{S} 99$ components are scaled according to the IMF used and the total mass of our simulated clusters is $10^{6} M_{\odot}$. We adopt a lower mass cutoff of $0.08 M_{\odot}$ and an upper mass cutoff of $100 M_{\odot}$. We have adopted solar metallicity for our analysis in the Antennae in accordance with metallicity measurements (Mengel 2001; Christopher 2008).

Assuming an IMF and an age, all stars below the PMS cutoff in the simulated cluster are assigned a standard star spectrum
(Meyer et al. 1998; Wallace \& Hinkle 1997) depending on their temperature, with an appropriate luminosity. The standards used are field dwarfs that cover a spectral range between B2 and M6. The spectra are scaled with the appropriate luminosity, co-added and weighted by the main sequence and post-main sequence contribution from S99.

\subsection{Nebular Emission}

In addition to stellar light, the near-infrared spectrum also contains nebular free-free and free-bound emission due to ionizing radiation emitted by the massive stars present in highmass clusters. S99 predicts that at very young ages up to $90 \%$ of the near-infrared luminosity of young SSC should be emitted in nebular mission. However, the free-free and free-bound components can be constrained directly through thermal radio data. Cluster 89/90 was observed by Neff \& Ulvestad (2000) at 4 and $6 \mathrm{~cm}$ (Table 1). Using the $4 \mathrm{~cm}$ flux, which is less susceptible to non-thermal contamination, we can estimate the Lyman continuum flux being emitted by the cluster in the $H$ and $K$ band in the following way. The number of Lyman continuum photons is related to the radio flux by the following formula (Condon 1992):

$$
\begin{aligned}
Q_{\mathrm{Lyc}} \geqslant & 6.3 \times 10^{52}\left(\frac{T_{e}}{10^{4} \mathrm{~K}}\right)^{-0.45} \times\left(\frac{v}{\mathrm{GHz}}\right)^{0.1} \\
& \times\left(\frac{F_{\text {nebular }}}{10^{27} \mathrm{erg} \mathrm{s}^{-1} \mathrm{~Hz}^{-1}}\right),
\end{aligned}
$$

where $T_{e}$ is the electron temperature. We assumed a value of $7500 \mathrm{~K}$ for $T_{e}$ which is a "typical" temperature assumed for Galactic UC H II regions and the same temperature assumed by Neff \& Ulvestad (2000). For cluster $89 / 90$, this results in $Q_{\text {Lyc }} \geqslant 8.46 \times 10^{52} s^{-1}$ which corresponds to the equivalent of $\geqslant 2300$ O5 stars powering the free-free and free-bound emission of the cluster. We then convert this to a nebular flux in the $H$ and $K$ band following:

$$
F_{\text {nebular }}=\left(\frac{c}{\lambda^{2}}\right) \times\left(\frac{\gamma_{\text {total }}}{\alpha_{B}}\right) \times Q_{\mathrm{Lyc}} .
$$

Here, $\alpha_{B}$ refers to the case $\mathrm{B}$ recombination coefficient and $\gamma_{\text {total }}$ is the continuous emission coefficient. The values used were adopted from Ferland (1980). With the assumption of 
Table 2

Best-fit Models

\begin{tabular}{lccrr}
\hline \hline \multicolumn{1}{c}{ Model Prescription } & Age (Myr) & $\alpha^{\mathrm{a}}$ & $M_{\text {old }} / M_{\text {young }}$ & $M_{\text {tot }}\left(M_{\odot}\right)^{\mathrm{b}}$ \\
\hline Single burst & 1 & -3.0 & $\ldots$ & $2 \times 10^{7}$ \\
Two bursts (Population A) & 1 & -2.35 & 0.04 & 1.40 \\
Two bursts (Population B) & 12 & -2.35 & 0.04 & $1.4 \times 10^{7}$ \\
\hline
\end{tabular}

Notes.

a Power-law slope of the IMF $\left(d N / d m \propto m^{-\alpha}\right)$.

b Flux due to best-fit model as compared with the total $K$-band flux of cluster 89/90.

a distance for the Antennae, this value can then be directly compared to the measured near-infrared flux for cluster $89 / 90$ in the $H$ band and the $K$ band (see Table 1). This results in $F_{\text {nebH }}=2.78 \times 10^{-13}$ and $F_{\text {nebK }}=1.98 \times 10^{-13} \mathrm{erg} \mathrm{s}^{-1}$ $\mu \mathrm{m}^{-1} \mathrm{~cm}^{-2}$ in cluster $89 / 90$. This corresponds to $19.1 \%$ and $23.5 \%$ of the total $\mathrm{H}$ - and $\mathrm{K}$-band flux from the cluster. This is the value we will use for the rest of the analysis as the nebular percentage contributing to the near-infrared emission in cluster 89/90. The slope of the free-free and free-bound emission in the near-infrared is $f_{f f} \propto \lambda^{-2}$. S99 assumes that every Lyman continuum photon emitted in the cluster is absorbed and reradiated into free-free or free-bound emission (case B). This assumption seems to overestimate the measured nebular emission in young SSC. We attribute this to the fact that the dust surrounding the hot stars in young clusters is likely clumpy. Indebetouw et al. (2006) have found that the nearinfrared spectral energy distributions of high-mass stars can vary by orders of magnitude depending on the clumpiness of the circumstellar material.

\section{APPLICATION TO CLUSTER 89/90 IN THE ANTENNAE}

We now model the spectrum of cluster 89/90 comparing the NIRSPEC spectrum to the results of the modeling routine described above. Figure 1 shows the $H$ - and $K$-band spectrum of the cluster with emission lines marked. The absorption lines are barely visible at this scale. Emission lines are not included in our model or our analysis. To model the spectrum of cluster 89/90, we first assume that the cluster represents one co-eval burst of star formation (see Section 4.1) and explore the IMF required to reproduce the spectrum. We also consider two separate bursts as the underlying population of the spectrum (see Section 4.2), since (1) the spatial scale covered by our spectrum is quite large with $\approx 50 \%$ of the clusters in the sample of Christopher (2008) revealed as multiple clusters in HST imaging and (2) the IMF required by a single coeval burst exceeds the Salpeter slope. Table 2 lists the best-fit model parameters for the two approaches.

\subsection{Single-age Burst}

To constrain the IMF required in cluster $89 / 90$ if the latetype absorption features are due to a single-age burst, we model clusters at ages of $0.3,1.3$, and 5 Myr. Above $6 \mathrm{Myr}$, red supergiants appear in clusters, and our single-burst model would no longer be appropriate due to the observed ratios of nearinfrared absorption lines in our spectrum (as explained in more detail in the Section 4.2). We found that the $5 \mathrm{Myr}$ models could not accurately reproduce the observed spectrum because of the rapid dimming of the PMS stars and these models are not included in the two-burst model for that reason. We use a single variable power law for the IMF with a break point at
$1 M_{\odot}$ with a variable slope below the break point and a Salpeter (1955) slope ( $\alpha=2.35$ in linear units) above. We vary the slope below $1 M_{\odot}$ by 0.5 dex between 0.0 and 4.0. To determine which models best fit the cluster spectrum, we performed a $\chi^{2}$ analysis comparing the model spectrum convolved to the resolution of the data and the data spectrum, which has been shifted to rest-frame wavelengths and normalized by fitting a fifth-order polynomial to the continuum. The nebular emission in the cluster is calculated as given above from the radio data of Neff \& Ulvestad (2000) and is independent of these assumptions. We included five spectral regions in our fit, which are marked in Figure 2. These five regions include late-type absorption lines that are seen in red supergiants as well as PMS stars. In the $\mathrm{H}$ band, there are numerous ${ }^{12} \mathrm{CO}$ transitions that are surface gravity sensitive and strong in red supergiants but weak in main sequence and PMS objects. Of these, we include the transitions at $1.62 \mu \mathrm{m}$ and $1.71 \mu \mathrm{m}$ in our fit. The $\mathrm{CO}(2-0) 2.29 \mu \mathrm{m}$ transition in the $K$ band is also surface gravity sensitive but is a prominent feature in all late-type objects. The $\mathrm{Ca}$ triplet (2.261, 2.263, and $2.117 \mu \mathrm{m}$ ) and the Na doublet (2.206 and $2.208 \mu \mathrm{m}$ ) in the $K$ band are strongest in stars below $3500 \mathrm{~K}$ $\left(\leqslant 0.5 M_{\odot}\right)$. In addition, we included the $\mathrm{Mg}$ I $1.71 \mu \mathrm{m}$ feature which is blended with one of the ${ }^{12} \mathrm{CO}$ features at $1.71 \mu \mathrm{m}$ and is strongest in stars between 0.5 and $1.5 M_{\odot}(3500-7000 \mathrm{~K})$. The presence of the $\mathrm{Mg}$ (solar-type stars) line in combination with the $\mathrm{Ca}, \mathrm{Na}$, and $\mathrm{CO}$ (cooler stars) features is what allows us to place constraints on the IMF (Meyer \& Greissl 2005). We then minimize $\chi^{2}$ of the difference between the model spectrum and the data. Here, the goodness of the fit is given by $\chi^{2}=$ $\sum_{i=0}^{n}\left(d_{i}-m_{i}\right) / \sigma_{i}^{2}$, where $d_{i}$ refers to the $i$ th data pixel and $m_{i}$ refers to the corresponding model point and $\sigma_{i}^{2}$ is the $\mathrm{S} / \mathrm{N}$ value at the $i$ th pixel. $\chi^{2}$ is reduced in the standard way by diving by the number of pixels in our absorption bands minus the degrees of freedom in our analysis. This process is described in more detail in the following section.

The best-fit model is plotted in Figure 2; and the model parameters are listed in Table 2. This single-age model does not match the depth of the surface gravity sensitive $\mathrm{CO}$ features well and the spectrum which best matches the data has $d N / d M$ $\propto m^{-3.0}$ or steeper. As this seems extraordinary, we consider an alternate hypothesis of two separate bursts of star formation at different ages.

\subsection{Two Separate Bursts}

We now attempt to reproduce the spectrum given two underlying bursts of star formation. Given that our 1" slit represents a physical scale of $\approx 90 \mathrm{pc}$ at the distance of the Antennae, this approach seems warranted. We model the spectrum as one young population containing PMS stars (hereafter, Population "A") in addition to an older population containing red supergiants (hereafter, Population "B"). We model these populations separately and then combine the two. Incorporating an older 

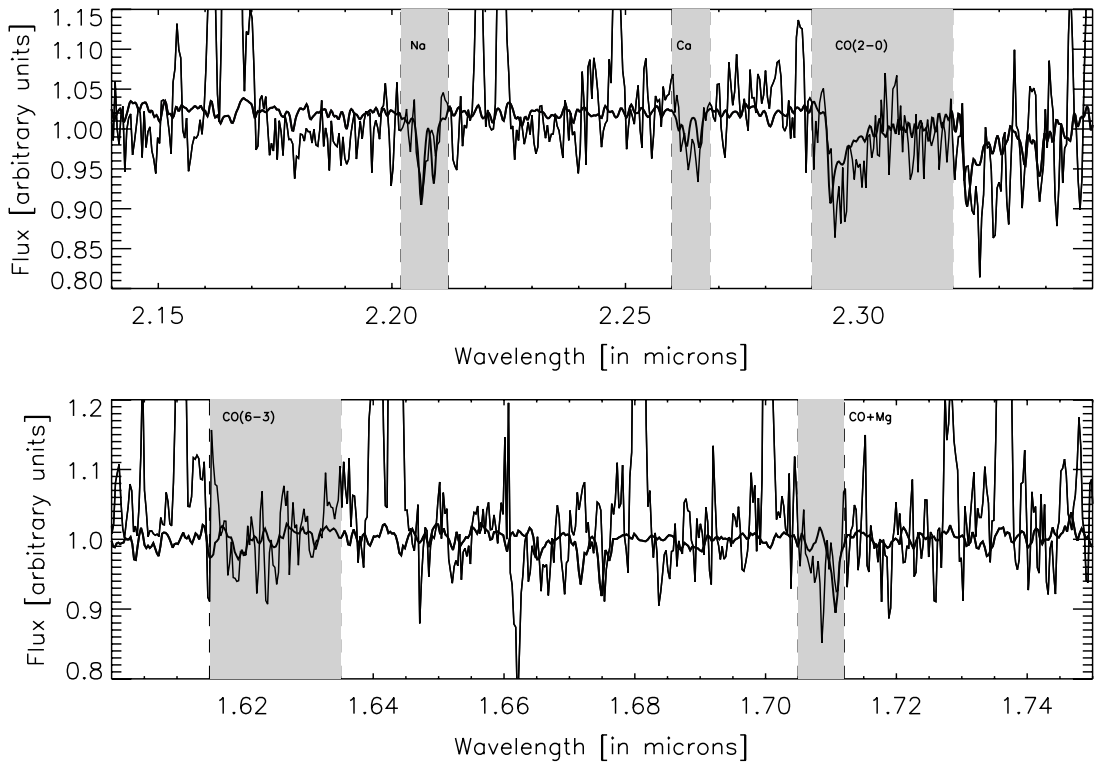

Figure 2. Best-fit single-age model overlaid over a normalized scaled spectrum of cluster $89 / 90$. The bands used in our analysis are marked. The emission lines as well as large parts of the continuum were not included in the modeling. The best-fit reduced $\chi^{2}=1.40$.

cluster containing supergiants is supported by the fact that the $H$-band spectrum of cluster $89 / 90$ contains ${ }^{12} \mathrm{CO}$ absorption lines which are much stronger in supergiants than in dwarfs and PMS stars (see Figures 2 and 4). However, Figure 3 shows that cluster 89/90 lies below supergiant standards when using an index that is surface gravity sensitive as function of temperature. Giants lie between supergiants and dwarfs in this index but are not included in the figure since giants do not appear until ages $\geqslant 100$ Myr. This index includes the surface gravity sensitive $\mathrm{CO}(2-0) 2.29 \mu \mathrm{m}$ feature as well as the Na I doublet and the $\mathrm{Ca}$ I triplet which are not strongly affected by surface gravity, though the ratio of the lines in the Na I doublet changes with $\log g$. For reference, supergiant sources have $\log g=0.0$ while dwarfs have $\log g=5.0-5.5$. Cluster 89/90 lies close to the dwarf locus in this index which indicates that the late-type absorption features in cluster $89 / 90$ cannot be caused solely by red supergiants, but must have an underlying PMS component. ${ }^{5}$ The presence of a young component is also supported by the emission lines seen in the spectrum of cluster $89 / 90$. The red supergiants have stronger late-type absorption lines but cannot match the observed surface gravity sensitive equivalent width ratios. A combination of red supergiants and late-type dwarfs however can reproduce both the surface gravity index as well as the overall depth of the absorption features. In our best-fit model, A and B have a flux ratio of $\approx 7$ to 1 , which is well reproduced by the location of Cluster 89/90 in Figure 3.

There are three variables we consider for each burst: mass, age, and IMF. The nebular emission in the cluster is again estimated from the radio data of Neff \& Ulvestad (2000). The spectrum of B is modeled using S99, with appropriate standard spectra (Meyer et al. 1998; Wallace \& Hinkle 1997) used for the red supergiants in the spectrum similar to A. The output of S99 includes the number of stars of each spectral type. For each supergiant of a given spectral type and temperature, we then use an appropriate spectral standard. The temperature scale used was

\footnotetext{
5 The values measured for Cluster $89 / 90$ for the equivalent widths in Figure 3 are $\mathrm{CO}(2-0)=14.84 \pm 3.29 \AA, \mathrm{Ca}=3.30 \pm 0.48 \AA$, and $\mathrm{Na}=4.19$ $\pm 0.63 \mathrm{~A}$. The $\mathrm{Ca}$ triplet and $\mathrm{Na}$ doublet bandpasses were chosen in accordance with Kleinmann \& Hall (1986) while the CO(2-0) bandpass was 2.293-2.318 $\mu \mathrm{m}$ with the continuum between 2.2887 and $2.2915 \mu \mathrm{m}$.
}

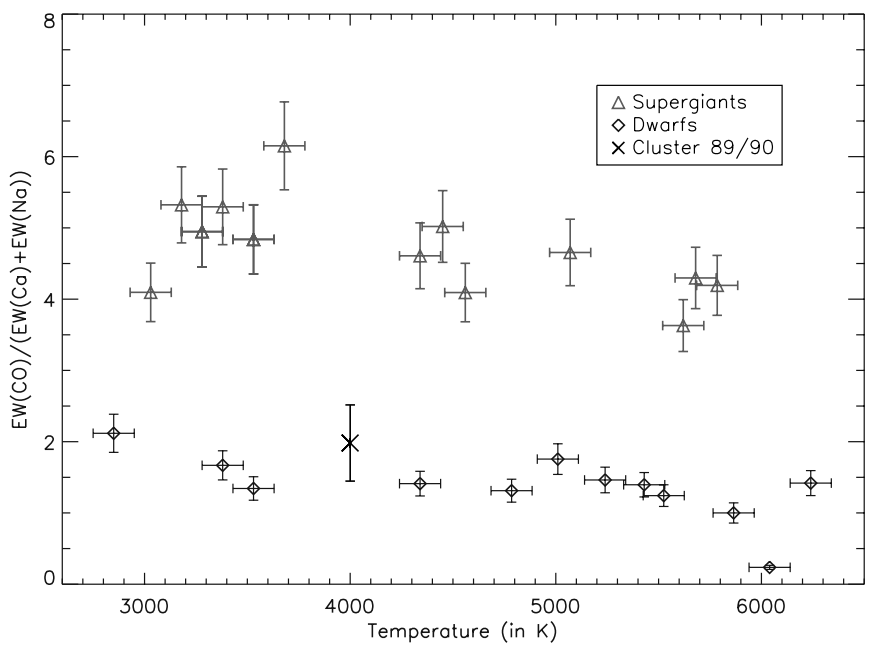

Figure 3. Plot of the ratio of equivalent widths of $\mathrm{CO}(3-1) 2.29 \mu \mathrm{m}, \mathrm{Ca}$ $2.26 \mu \mathrm{m}$, and $\mathrm{Na} 2.20 \mu \mathrm{m}$ absorption features in supergiant and dwarf standards. Overplotted is cluster 89/90 at an arbitrary temperature. Errors were estimated using standard error propagation. This shows the clear separation between supergiants and dwarfs in this index. Cluster 89/90 lies much closer to the dwarf locus rather than the supergiant locus. This indicates that a supergiant population cannot be solely responsible for the absorption lines present in the spectrum.

adapted from the cool supergiant temperature scale by Levesque et al. (2005).

\subsubsection{Model Parameters}

According to S99, the first supergiants in a star cluster appear around 6 Myr though a significant population does not appear before 7 Myr. Late-type supergiants disappear at an age of $\approx 30$ Myr. Thus, we can roughly constrain the age of $\mathrm{B}$ to between 7 and 30 Myr. In the $H$ band, cool stars possess a ${ }^{12} \mathrm{CO}$ absorption line which blends with the $1.71 \mu \mathrm{m} \mathrm{Mg}$ line in the $H$ band (see Figure 2). This feature is strongly surface gravity sensitive and very weak in dwarfs. This blend is observed in Cluster 89/90 giving additional credence to the fact that red supergiant features are present in our spectrum. We considered models in $3 \mathrm{Myr}$ steps. 

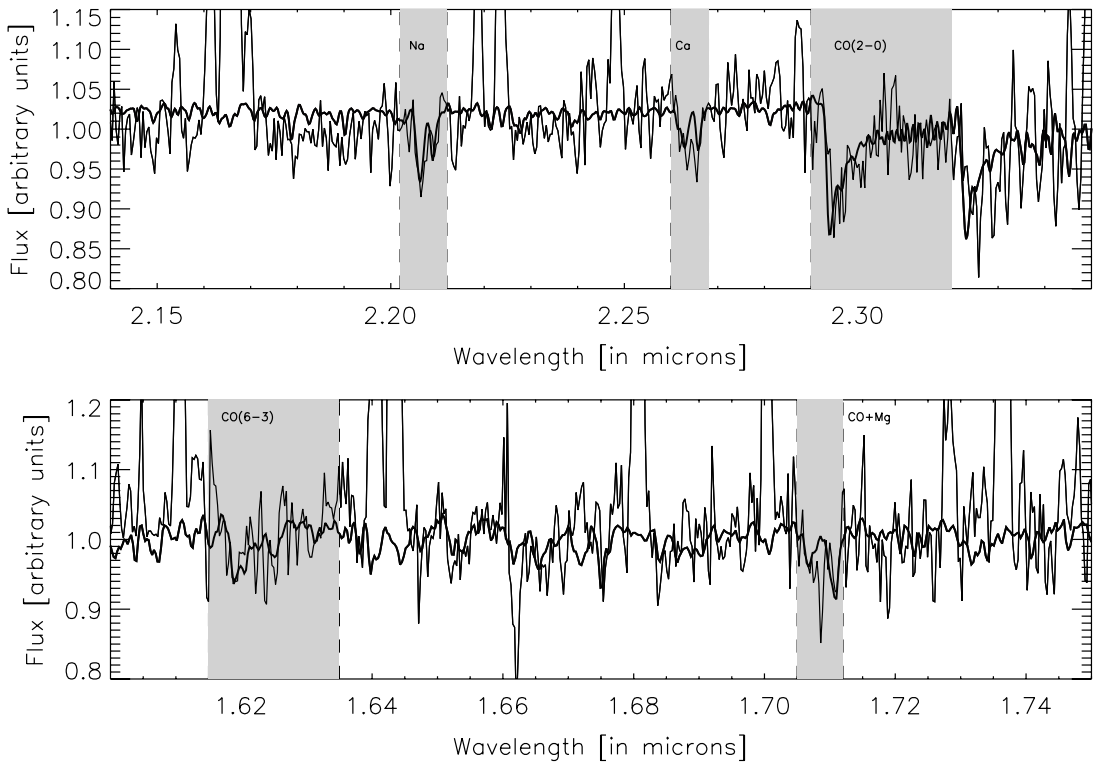

Figure 4. Best-fit two-burst model overlaid over a normalized scaled spectrum of cluster $89 / 90$. The bands used in our analysis are marked. The emission lines as well as large parts of the continuum were not included in the modeling. The best-fit reduced $\chi^{2}=1.23$.

For A, the goal was not to constrain the age accurately but to assure that the best fit was consistent across different ages. Changing results with age would have limited the usefulness of our analysis method, since we do not have an independent constraint on the age of A except for an upper limit on the age of $\leqslant 5$ Myr. We considered models of $0.3,1$, and $3 \mathrm{Myr}$.

We vary the mass ratio of the two populations between zero and one in steps of 0.01 below $M_{\text {old }} / M_{\text {young }}=0.2$ and in steps of 0.1 above 0.2 . We cannot directly constrain the total mass of the cluster, because the overall flux of the spectral model depends on the mass ratio of the best-fit model. After we have determined the best spectral model fit, it is possible to constrain the mass through broadband magnitudes. There is no way to directly constrain the IMF of B since we only trace a very small mass range of stars in the population in our spectrum, the red supergiants. Thus, the IMF of B is assumed to be the same as that of A. For A, we have allowed the IMF to vary as a broken power law with a Salpeter slope above $1 M_{\odot}$ and a varying slope below by 0.5 dex between 0.0 and 2.0 and also including 2.35 . We did not attempt varying the break point in the IMF or introduce a low-mass cutoff. There is some evidence that one could expect the Jeans mass to vary in SSCs which would influence the break point of the IMF (Larson 2005). The limitations of this particular data set, due to a possible overlap of two star clusters, make such a study not feasible.

\subsubsection{Analysis}

We again perform a $\chi^{2}$ analysis between the model spectra and the data including the same spectral regions as in 4.1. To assess the $\mathrm{S} / \mathrm{N}$ of the observed spectrum at each spectral region, we fit the continuum of the spectrum around each of the absorption bands and measured the rms noise around the fit. We estimate the average $\mathrm{S} / \mathrm{N}$ of the spectrum to be $\approx 30$. We ran models in all permutations of parameters described in the previous section and determined the best-fit model (with the lowest $\chi^{2}$ ), which is shown in Figure 4.

Given the large grid of models, we tried to constrain the acceptable range of values for each model parameter in the following way. First, we determined the probability of each model given by $P_{i} \propto e^{-\chi_{i}{ }^{2}}$ and normalized so that $\sum_{i=0}^{n} P_{i}=$ 1 . We then plot the probability separately for each variable (see Figure 5), where each point in the plot represents the sum of the probabilities over the whole range of the other two variables. We also assessed the probabilities in a Monte Carlo way. We added a noise spectrum given by a resampled subtraction of the data and the best-fit model to the cluster spectrum and determined the best fit for each newly realized resampled spectrum. We performed this routine 10,000 times and determined the probability that a particular set of parameters is determined as the best-fit model. The results from the Monte Carlo simulations agreed well with the probabilities determined from the $\chi^{2}$ values alone.

\section{RESULTS}

\subsection{Best-fit Models}

We now discuss the implications of the ranges of parameters constrained by our modeling (see Table 2). The three plots in Figure 5 show the probability distribution of the age of the old population, mass ratio of the two bursts, as well as the slope of the IMF below $1 M_{\odot}$. Each plot shows the probability distribution at the three different ages of A to make sure that the results are consistent over a range of ages. Panel 1 shows that the older population has a most probable age of $12 \mathrm{Myr}$. Within $90 \%$ confidence limits, the models are consistent with an age between 6 and $18 \mathrm{Myr}$ for the old population. It should be noted that a population containing no supergiants is ruled out as can be seen by the fact that an age of $1 \mathrm{Myr}$ and $3 \mathrm{Myr}$ for B have zero probability. This coarse age constraint is not surprising since very little differentiates spectra of red supergiants in the near-infrared besides the overall strength of late-type absorption lines.

The plot of mass ratios shows the cumulative probability with respect to $M_{\text {old/young }}$. The mass ratio strongly favors A dominating in mass by a factor of $\geqslant 5$ over $\mathrm{B}$. The best-fit model comparison of the $K$-band flux with the $K$-band broadband flux of the cluster yields a total mass of $1.4 \times 10^{7} M_{\odot}$ down to the hydrogen burning limit. If we assume that the total mass is $1.4 \times 10^{7} M_{\odot}$ then the total mass of A is $\geqslant 1.2 \times 10^{7} M_{\odot}$, while 

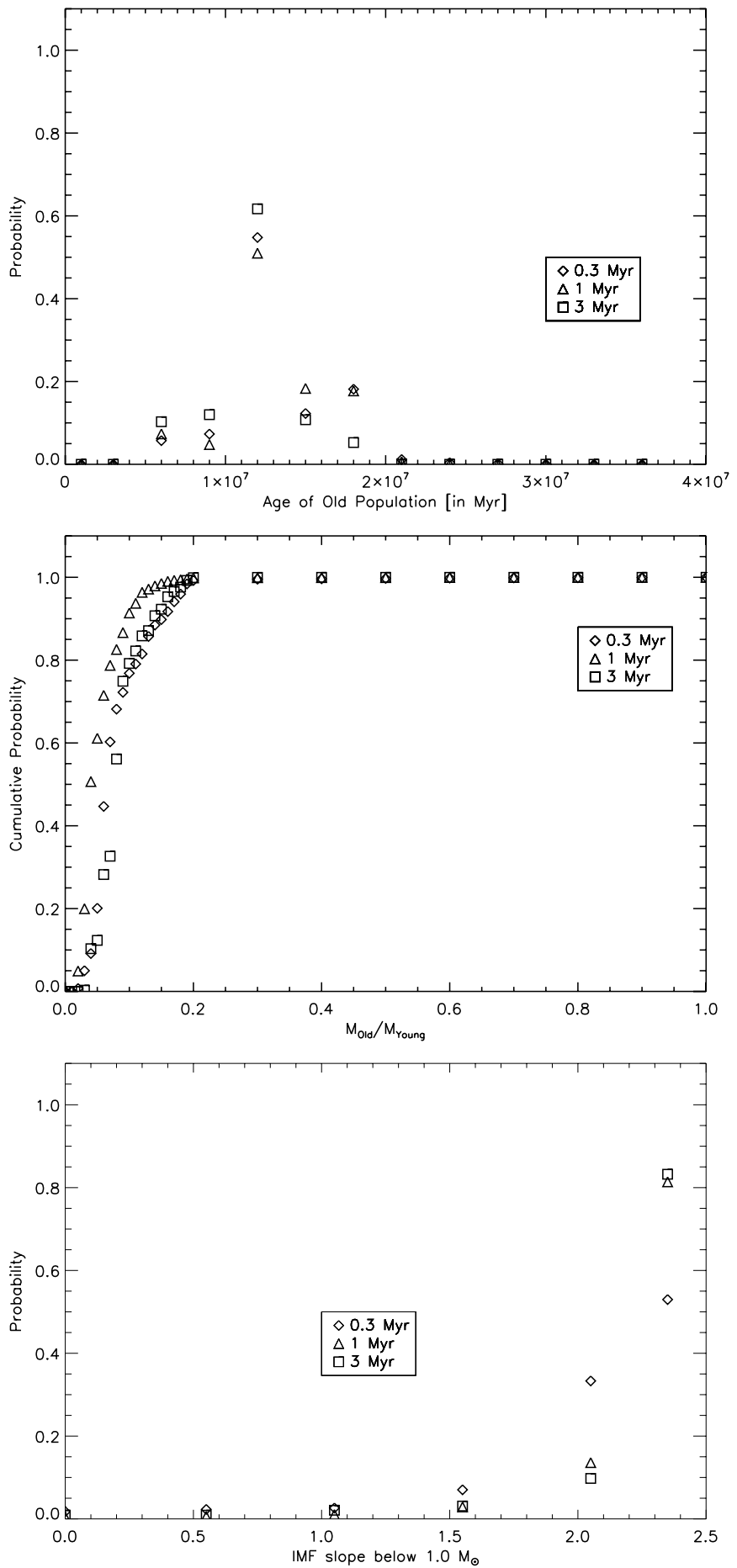

Figure 5. Plot of probabilities vs. age (top panel), mass ratio (middle panel), and IMF (bottom panel) for our models. Each plot includes separate data points for all three young burst ages to make certain that our results are not age dependent. Each plot point in a panel represents a summation over all the values of the other two variables and for each panel the probability has been normalized. For clarity, the mass ratio is shown as the cumulative probability. Both the IMF and the age plots show well-constrained values at the resolution of the models. The mass ratio is less tightly constrained.

the mass for $\mathrm{B}$ is $\leqslant 2 \times 10^{6} M_{\odot}$. The best fit indicates a mass of B of $5 \times 10^{5} M_{\odot}$ and a ratio of $M_{\text {old }} / M_{\text {young }}=0.04$. The total mass estimate agrees well with that calculated by Gilbert et al. $\left(2000 ; 1.6 \times 10^{7} M_{\odot}\right)$ using the Lyman continuum flux of cluster $89 / 90$. Thus, the young component of cluster $89 / 90$ likely is one of the most massive young star clusters which formed in the Antennae galaxies. The $90 \%$ confidence limit for the mass ratio is roughly $0.02-0.12$ though this value changes slightly between different ages of A. Thus, we have clearly detected the PMS in the cluster. A mass ratio of 0 and therefore $M_{\text {old }}=0$ is ruled out as well as a mass ratio $\geqslant 0.12$ and thus no PMS contribution.

The probability for the IMF of A rises strongly toward steeper slopes with the probability being highest for a Salpeter slope below $1 M_{\odot}$. However, the spectrum is formally consistent with a slope down to a power-law slope of 1.5 within a $90 \%$ confidence limit. This result is consistent across all ages of the young population. A top-heavy IMF weighted more heavily toward high-mass stars than a Kroupa (2001) IMF has often been cited as expected for SSC clusters, and the best remaining evidence of an unusual IMF in an SSC (M82-F) indicates a top-heavy IMF. Our result is consistent with a normal Galactic IMF (Covey et al. 2008) as well as a Salpeter IMF. A lowmass cutoff in this cluster is ruled out since PMS objects in the young burst below $1.0 M_{\odot}$ are required to produce the observed spectrum. We did not include any slopes above Salpeter as the goal of this study was not to make claims of extraordinary IMF results within the limited data this cluster offers. In addition, the best-fit model for the two-burst model at a Salpeter slope shown in Figure 4 is a very good fit to the data whereas for the single burst even a Salpeter slope did not fit the data well. This is independent of the probability curve shown, which was shown to illustrate the overall trends of the models rather than the quality of the overall fits. Thus, we feel, it is reasonable to set the cutoff at the Salpeter slope even though the IMF probability is still rising.

\subsection{Caveats}

Our model has many components and therefore degeneracies exist between its different subsets. One such degeneracy exists between the assumed age of the PMS objects and the IMF slope. Since PMS objects grow fainter as they age, one might erroneously assign an IMF that is too flat to a $1 \mathrm{Myr}$ cluster that is really $3 \mathrm{Myr}$ old. However, Figure 5 shows that our IMF results are very similar at different assumed ages for the young burst, and thus we do not expect this to be a problem. Another concern is that the calculated nebular emission is wrong and this might affect the results of our models. We varied the input nebular continuum by $50 \%$ in both directions which did not change our results substantially.

In addition, there are limits to the inputs of our models. Our standards are field dwarfs with high surface gravity, while PMS objects are young and have lower surface gravity than dwarfs. The surface gravity of PMS objects is generally between $\log g$ $=3.0$ and 4.2 (Gorlova et al. 2003), while dwarfs are generally have $\log g=5.0-5.5$. This would increase the depth of the $\mathrm{CO}$ absorption features in our spectrum. Obtaining spectral standards of young PMS objects is difficult because the largest nearby sample is found in relatively distant young clusters, making it observationally expensive to obtain a complete high $\mathrm{S} / \mathrm{N}$ sample in the near-infrared. Since we used spectral standards to model our spectra rather than synthetic spectra, our coverage of individual spectral types is not complete. This might cause errors in the depth of the absorption features of our model spectra due to binning the stars into spectral types for which spectral coverage exists. However, this effect is likely small compared to the error introduced by the usage of dwarf rather than PMS standards. Since synthetic spectra struggle to accurately model low-mass young PMS stars (Doppmann et al. 
2003), dwarf standards currently remain the best option to accurately model the near-infrared spectra of SSC. However, PMS stars generally lie closer to field dwarfs than supergiants in surface gravity (Gorlova et al. 2003); and it is thus likely that even including PMS standards would not remove the need of having a supergiant component in our models. The presence of veiling due to disks around young stars can dilute the absorption features in near-infrared spectra. We do not have an independent method of quantifying the affect veiling has on our data since we are modeling a whole star cluster, but we expect the effect to be minimized by the use of line ratios. Finally, in some very young objects, $\mathrm{CO}$ features can be seen in emission due to the presence of a disk (e.g., Blum \& McGregor 2008). We do not observe evidence for this in our spectrum.

\section{DISCUSSION}

\subsection{Previous Work}

We have shown that it is possible to directly detect PMS stars in unresolved SSC as well as place some constraints on the underlying IMF and age of the cluster. Substantial work has been done studying the IMF of SSC through measurements of mass-to-light ratios, including in the Antennae. Mengel et al. (2002) measured mass-to-light ratios in the $K$ band for a number of clusters in NGC 4038/9 and found variations indicative of IMF variations in the clusters. To measure mass-to-light ratios in clusters younger than $\approx 30 \mathrm{Myr}$ requires the ability to constrain velocity dispersions through strong $\mathrm{CO}$ absorption bands that exist in clusters dominated by red supergiants. Bastian et al. (2006) used UV spectra of clusters in two merger remnants with ages of more than $300 \mathrm{Myr}$ to measure their mass-to-light ratios and found them to be consistent with a Kroupa IMF. Similarly, Larsen et al. (2004) and Larsen \& Richtler (2004) measured mass-to-light ratios in older clusters through optical spectroscopy and found them to be consistent with a Kroupa IMF. This dichotomy: young clusters with varying mass-tolight ratios and older cluster with mass-to-light ratios consistent with normal IMFs provide evidence that something other than IMF variations are the cause of varying mass-to-light ratios in young SSC or that perhaps SSC with unusual IMFs get disrupted preferentially. This work shows an avenue for providing more concrete measurements of IMFs in SSC than mass-to-light measurements can deliver though this data set is not the ideal application of our method.

Work on modeling the integrated spectra of young clusters has up until now been done mostly in the UV and optical (e.g., Tremonti et al. 2001) because of the strong signatures of massive stars at these wavelengths. The near-infrared is ideally suited to detect the low-mass stellar content of the clusters as well as evolved giants and supergiants. Recently, Lançon et al. (2008) have attempted to use simple stellar population models to constrain ages of star clusters in M82 in the nearinfrared. Brandl et al. (2005) attempted to use near-infrared colors of clusters in the Antennae in combination with thermal radio images to constrain their properties and found them to be uncorrelated. However, Antennae clusters are not ideal objects to study young clusters in detail. They are distant enough that multiple stellar populations of different ages may be treated as unresolved point sources. We have shown that it is possible to constrain the properties of young star clusters through nearinfrared spectroscopy. Varying nebular emission due to the ionizing radiation emitted by hot stars provides an additional complication in the understanding of the youngest clusters. The total amount of free-free and free-bound emission in young clusters is easiest to constrain empirically, because it should depend strongly on the formation environment of the cluster. More work is needed studying nearby SSC in the near-infrared to understand whether near-infrared images and spectroscopy can accurately predict the ages and masses of these clusters.

\subsection{Scales of Star Formation}

What can cluster $89 / 90$ tell us about the scales of star formation in the Antennae galaxies as well as locally? The spectrum of our cluster likely contains two separate underlying populations with an age spread between 6 and $18 \mathrm{Myr}$ and a mass ratio $M_{\text {old }} / M_{\text {young }} \leqslant 0.2$. At the distance of the Antennae, our $1^{\prime \prime}$ slit covers a region of $93 \mathrm{pc}$. This region likely contains two massive clusters, one with a mass of order $10^{7} M_{\odot}$ and the other $10^{6} M_{\odot}$. It is possible that the older cluster started out at a higher mass and lost a large fraction of its mass due to dispersion into the field. How does this compare to sites of massive star formation we can study in detail?

No young $10^{6} M_{\odot}$ clusters exist in local group galaxies. However, massive sites of star formation (up to roughly $10^{5} M_{\odot}$ ) are accessible in both the Milky Way and the LMC. Grebel \& Chu (1999) present detailed observations of Hodge 301, a cluster in the 30 Doradus star-forming complex. Hodge 301 is between 20 and 25 Myr old and lies at a distance of $3^{\prime}(\approx 44 \mathrm{pc})$ from the young center of 30 Doradus, R136. It is estimated to have been relatively massive with an initial mass of up to $6000 M_{\odot}$ though less massive than R136 ( $\left.\approx 30,000 M_{\odot}\right)$. In the Galaxy, NGC 3603 and the Arches cluster, two of the most massive star-forming regions in the Milky Way do not appear to have similar older star formation complexes in their proximity, though both have isolated red supergiants in their environments. Melo et al. (2005) found the typical separation of young SSCs in M82 to be $\approx 12 \mathrm{pc}$ though the equivalent age spread of the clusters is not given. Christopher (2008) found that half of their Antennae NIRSPEC cluster sample with 1" resolution contained more than one star cluster which would indicate a larger typical star cluster separation than in M82. Christopher (2008) also found some sources in their Antennae sample with no obvious cluster superposition which contained emission lines as well as $\mathrm{CO}$ band heads simultaneously. This raises the question whether some SSCs have non-instantaneous bursts of star formation over the size of the cluster and about the magnitude of this age spread.

Cluster 89/90 illustrates the need to study massive starforming regions in detail at a distance where the individual cluster complexes can be resolved from each other. Neff \& Ulvestad (2000) found radii of $\approx 3 \mathrm{pc}$ for the thermal radio sources in NGC 4038/9. This distance corresponds to 0'.03 in the Antennae. If we assume a typical star cluster separation of $12 \mathrm{pc}$, the distance at which a $1^{\prime \prime}$ spectrum can resolve this distance is $\approx 2.5 \mathrm{Mpc}$. More distant galaxies make ideal targets for observations with adaptive optics (AO) equipped with integral field unit (IFU) spectrographs.

\section{SUMMARY AND CONCLUSIONS}

We have modeled the integrated properties of a massive young cluster in the Antennae through its $H$ - and $K$-band spectrum. Our models use a combination of Starburst99, PMS tracks, and field dwarf spectral standards. We find that the integrated spectrum likely represents two separate star clusters with an age spread of 6-18 Myr with a mass ratio of $M_{\text {old }} / M_{\text {young }} \leqslant 0.2$ and a total mass of $\approx 10^{7} M_{\odot}$. The IMF in the young cluster is best fit by a 
Salpeter (1955) slope down to the hydrogen burning limit though the cluster IMF is formally consistent with a Chabrier (2003) IMF. We find no evidence of a low-mass cutoff in the cluster. Thus, we have for the first time directly detected low-mass PMS stars in a young extragalactic SSC.

We thank the referee for helpful comments and suggestions that improved the content and clarity of the manuscript. The data presented here were obtained with the W. M. Keck Observatory, which is operated as a scientific partnership among the California Institute of Technology, the University of California, and the National Aeronautics and Space Administration. The authors wish to recognize and acknowledge the very significant cultural role and reverence that the Mauna Kea summit has always had within the indigenous Hawaiian community. We are most fortunate to have the opportunity to conduct observations from this mountain. This publication makes use of data products from the Two Micron All Sky Survey, which is a joint project of the University of Massachusetts and the Infrared Processing and Analysis Center/California Institute of Technology, funded by the National Aeronautics and Space Administration and the National Science Foundation. M.R.M. gratefully acknowledges the support of a Cottrell scholar award from the Research Corporation.

\section{REFERENCES}

Anders, P., de Grijs, R., Fritze-v., Alvensleben, U., \& Bissantz, N. 2004, MNRAS, 347, 17

Bastian, N., Saglia, R. P., Goodfrooij, P., \& Kissler-Patig, M. 2006, A\&A, 448, 881

Blum, R., \& McGregor, P. J. 2008, AJ, 135, 1708

Brandl, B., et al. 2005, ApJ, 635, 280

Chabrier, G. 2003, PASP, 115, 763

Christopher, M. 2008, PhD thesis, California Institute of Technology

Condon, J. J. 1992, ARA\&A, 30, 575

Covey, K. R., et al. 2008, AJ, 136, 1778

Doppmann, G. W., Jaffe, D. T., \& White, R. J. 2003, AJ, 126, 3043

Ferland, G. J. 1980, PASP, 92, 596

Gilbert, A., et al. 2000, ApJ, 533, 57

Goodwin, S., \& Bastian, N. 2006, MNRAS, 373, 752

Gorlova, N., Meyer, M. R., Liebert, J., \& Rieke, G. H. 2003, ApJ, 593, 1074
Grebel, E. K., \& Chu, Y.-H. 1999, AJ, 119, 787

Indebetouw, R., Whitney, B. A., Johnson, K. E., \& Wood, K. 2006, ApJ, 636, 362

Kleinmann, S. G., \& Hall, D. N. B. 1986, ApJS, 62, 501

Kroupa, P. 2001, MNRAS, 322, 231

Lançon, A., Gallagher, J. S., Mouhine, M., Smith, L. J., Ladjal, D., \& de Grijs, R. 2008, A\&A, 486, 165

Larsen, S. S., Brodie, J., \& Hunter, D. A. 2004, AJ, 128, 2295

Larsen, S. S., \& Richtler, T. 2004, A\&A, 427, 495

Larson, R. 2005, MNRAS, 359, 211

Leitherer, C. 2001, in ASP Conf. Ser. 245, Astrophysical Ages and Times Scales, ed. N. M. T. von Hippel, C. Simpson, \& N. Manset (San Francisco, CA: ASP), 390

Leitherer, C., et al. 1999, ApJS, 123, 3

Levesque, E., Massey, P., Olsen, K. A., Plez, B., Josselin, E., Maeder, A., \& Meynet, G. 2005, ApJ, 628, 973

Massey, P., \& Hunter, D. A. 1998, ApJ, 493, 180

McCrady, N., Graham, J. R., \& Vacca, W. D. 2005, ApJ, 621, 278

McLean, I. S., et al. 1998, Proc. SPIE, 3354, 566

Melo, V. P., Munoz-Tunon, C., Maiz-Apellaniz, J., \& Tenorio-Tagle, G. 2005, ApJ, 619, 270

Mengel, S. 2001, PhD thesis, Ludwig-Maximilians-University Munich

Mengel, S., Lehnert, M. D., Thatte, N., \& Genzel, R. 2002, A\&A, 383, 137

Meyer, M. R., Adams, F. C., Hillenbrand, L. A., Carpenter, J. M., \& Larson, R. B. 2000, in Protostars and Planets IV, ed. V. Mannings, A. P. Boss, \& S. S. Russell (Tucson, AZ: Univ. Arizona Press), 121

Meyer, M. R., Edwards, S., Hinkle, K. H., \& Strom, S. E. 1998, ApJ, 508, 397

Meyer, M., \& Greissl, J. 2005, ApJ, 630, 177L

Mirabel, I. F., et al. 1998, A\&A, 333, 1

Neff, S. G., \& Ulvestad, J. S. 2000, AJ, 120, 670

Salpeter, E. E. 1955, ApJ, 121, 161

Siess, L., Dufour, E., \& Forestini, M. 2000, A\&A, 358, 593

Smith, L. J., \& Gallagher, J. S. 2001, MNRAS, 326, 1027

Snijders, L., van der Werf, P. P., Brandl, B. R., Mengel, S., Schaerer, D., \& Wang, Z. 2006, ApJ, 648, 25L

Steidel, C. C., Giavalisco, M., Pettini, M., Dickinson, M., \& Adelberger, K. L. 1996, ApJ, 462, L17

Tremonti, C. A., Calzetti, D., Leitherer, C., \& Heckman, T. M. 2001, ApJ, 555 , 322

Vazquez, G. A., \& Leitherer, C. 2005, ApJ, 621, 695

Wallace, L., \& Hinkle, K. 1997, ApJS, 111, 445

Wang, Z., et al. 2004, ApJS, 154, 193

Whitmore, B. C., \& Schweizer, F. 1995, AJ, 109, 960

Whitmore, B. C., Zhang, Q., Leitherer, C., Fall, S. M., Schweizer, F., \& Miller, B. W. 1999, AJ, 118, 1551

Whitmore, B. C., \& Zhang, Q. 2002, AJ, 124, 1418 